\title{
Synaptic Transmission between Dorsal Root Ganglion and Dorsal Horn Neurons in Culture: Antagonism of Monosynaptic Excitatory Postsynaptic Potentials and Glutamate Excitation by Kynurenate ${ }^{1}$
}

\author{
C. E. JAHR AND T. M. JESSELL ${ }^{2}$ \\ Department of Neurobiology, Harvard Medical School, Boston, Massachusetts 02115
}

\begin{abstract}
Intracellular recording techniques have been used to provide information on the identity of excitatory sensory transmitters released at synapses formed between dorsal root ganglion (DRG) and dorsal horn neurons maintained in cell culture. Explants of embryonic rat DRG were added to dissociated cultures of embryonic dorsal horn neurons and synaptic potentials were recorded intracellularly from dorsal horn neurons after DRG explant stimulation. More than $80 \%$ of dorsal horn neurons within $1 \mathrm{~mm}$ of DRG explants received at least one fast, DRG-evoked, monosynaptic input. In the presence of high divalent cation concentrations, the acidic amino acid receptor agonists, L-glutamate, kainate, and quisqualate excited all dorsal horn neurons which received a monosynaptic DRG neuron input, whereas aspartate and $\mathbf{N}$ methyl-D-aspartate (NMDA) had little or no action. Several compounds reported to antagonize the actions of acidic amino acids were tested for their ability to block DRG-evoked synaptic potentials and glutamate-evoked responses in dorsal horn neurons.

2-Amino-5-phosphonovalerate, a selective NMDA receptor antagonist, was relatively ineffective at antagonizing DRGevoked synaptic potentials and glutamate-evoked responses. In contrast, kynurenate was found to be a potent antagonist of amino acid-evoked responses and of synaptic transmission at all DRG-dorsal horn synapses examined. The blockade of synaptic transmission by kynurenate appeared to result from a postsynaptic action on dorsal horn neurons. These findings indicate that glutamate, or a glutamate-like compound, but not aspartate, is the excitatory transmitter that mediates fast excitatory postsynaptic potentials at the DRG-dorsal horn synapses examined in this study.
\end{abstract}

The transmission of sensory information at primary afferent synapses in the spinal cord involves the release of sensory transmitter(s) that elicits both fast and slow excitatory postsynaptic potentials (EPSPs) in postsynaptic spinal neurons (Eccles, 1961; Urban and

Reccived November 19, 1984; Revised January 21, 1985;

Accepted January 22, 1985

We wish to thank Ur. K. Yoshioka for helptul discussions and Drs. P. $R$. Adams, J. Dodd, A. Willard, and K. Yoshioka for critical reading of the manuscript. This work was supported in part by National Institutes of Health Grants NS 17369, NS 20116, NS 21419, and NS 07051, and by the McKnight Foundation, a Dupont Faculty Award, and the Muscular Dystrophy Association.

${ }^{2}$ To whom correspondence should be addressed.
Randic, 1984). Several lines of evidence suggest that the fast EPSPs in spinal cord neurons evoked by primary afferent stimulation are mediated by L-glutamate or by compounds with similar postsynaptic actions. Iontophoretic and pressure applications of L-glutamate depolarize the majority of mammalian spinal neurons in vivo and in vitro (Ransom et al., 1977b; Watkins and Evans, 1981; Salt and Hill, 1983) with a reversal potential (Mayer and Westbrook, 1984) similar to that of the EPSP evoked by dorsal root ganglion (DRG) neuron slimulation (Enybery and Marshall, 1979; Finkel and Redman, 1983; MacDonald et al., 1983). Biochemical analysis has demonstrated a higher concentration of L-glutamate in dorsal than in ventral roots (Roberts et al., 1973) and has provided evidence for release of endogenous $\mathrm{L}$-glutamate from regions of the CNS containing primary allerent terminals (Robuerts, 1974; Takeuchi et al., 1983). Moreover, L-glutamate-binding sites are found in high density in the superficial dorsal horn of rat spinal cord (Greenamyre et al., 1984) suggesting that amino acids may function as sensory transmitters released from cutaneous afferents.

Direcl conlimmalion of the role of acidic amino acids as primary sensory transmitters is still lacking. In studies with intact spinal cord preparations it has been difficult to distinguish the direct effects of exogenously applied amino acid transmitter candidates. Moreover, pharmacological studies have provided evidence for at least three arnirio acid receptor subtypes, each of which can be activated by L-glutamate (Watkins and Evans, 1981; Foster and Fagg, 1984). Selective ligands are available for only one of the receptor subtypes: $\mathrm{N}$-methyl-D-aspartate (NMDA) is a selective agonist and 2-amino-5phosphonovalerate (APV) is a relatively selective antagonist at the NMDA subclass of receptor (Davies et al., 1981a). The ion channel opened by NMDA receptor occupation is subject to a voltagedependent blockade in the presence of micromolar concentrations of magnesium ions (Ault et al., 1980; Mayer et al., 1984; Nowack et al., 1984) thus providing a second means of distinguishing this receptor subtype. The existence of two additional classes of arrino acid receptors has been proposed on the basis of pharmacological studies with the rigid amino acid analogues quisqualic acid and kainic acid (Watkins and Evans, 1981; Foster and Fagg, 1984). These two receptors are more difficult to distinguish from each other. They are not blocked by magnesium ions or by APV bul may be blocked differentially by $\gamma$-D-glutamylglycine and piperidine dicarboxylic acid (Davis and Watkins, 1981). These compounds, however, appear to be relatively nonselective since they also block the actions of amino acids at NMDA receptor sites (Francis et al., 1980; Davies el al., 1981b).

To provide more direct information on the identity of primary sensory transmitters and to characterize the postsynaptic receptors that mediate fast EPSPs at sensory synapses, we examined the actions of excitatory amino acid receptor ligands at afferent synapses in cell culture. Intracellular recording was used to identify dorsal horn neurons that receive monosynaptic DRG input and to 
characterize the sensitivity of these neurons to excitatory amino acid agonists. Several excitatory amino acid antagonists have been examined, and kynurenate (Perkins and Stone, 1982) was identified as a potent antagonist of synaptic transmission between DRG and dorsal horn neurons. Kynurenate has been reported to antagonize EPSPs in hippocampal pyramidal neurons and ventral root potentials in the rat (Ganong et al., 1983) and frog (Elmslie and Yoshikami, 1983) spinal cord. Our results suggest that L-glutamate or a closely related compound, but not L-aspartate, is likely to be the transmitter released at the sensory synapses examined in the present study.

\section{Materials and Methods}

Dorsal horn neurons were obtained from the dorsal half of spinal cords of 15-day rat embryos. After removal of the dura, the dorsal spinal cord was chopped in two directions at $200-\mu \mathrm{m}$ intervals and then incubated with trypsin $(0.025 \%)$ and DNase $(80 \mu \mathrm{g} / \mathrm{ml})$ (Yamamoto et al., 1981; Jahr and Jessell, 1983) for $15 \mathrm{~min}$ at $37^{\circ} \mathrm{C}$. The dorsal spinal tissue was dissociated into a single cell suspension by trituration with a fire-polished Pasteur pipette. Aliquots of the cell suspension were plated onto collagen-coated $35-\mathrm{mm}$ tissue culture dishes containing a confluent monolayer of non-neuronal cells (predominately astrocytes) derived from 15- to 17-day embryonic rat spinal cord. DRGs were dissected from 16- to 17-day rat embryos, incubated overnight in arabinosylcytosine $\left(10^{-5} \mathrm{M}\right)$ to suppress the proliferation of nonneural cells, and added to cultures of dorsal horn neurons which had been plated 1 to 17 days earlier. Cultures were fed every 2 to 3 days by replacing half the volume of medium (Ham's F12 supplemented with $5 \%$ heat-inactivated rat serum, $4 \%$ 17-day embryonic rat extract, $44 \mathrm{~mm}$ glucose, $2 \mathrm{~mm}$ glutamine, $1 \mathrm{~mm} \mathrm{CaCl}$, $1 \%$ Eagle's minimum essential medium $\times 100$ stock vitamins, $50 \mathrm{JU} / \mathrm{ml}$ of peniccilin/streptomicin, $1 \mu \mathrm{g} / \mathrm{ml}$ of purified $7 \mathrm{~S}$ nerve growth factor).

Intracellular recordings were made from dorsal horn and DRG ncurons grown in culture for 4 to 12 weeks. Microelectrodes were filled with $2 \mathrm{M}$ potassium methylsulfate or $3 \mathrm{M}$ potassium acetate and had resistances of 100 to 200 megohms. DRG explants were stimulated with a metal concentric bipolar electrode, the central core of which was buried in the explant. Square pulses of 1 to $20 \mathrm{~V}$ and 100 to $200 \mathrm{msec}$ duration were used to stimulate the explants. Intracellular recordings were stored on FM tape and averaged on a Nicolet 1074 signal averager. Excitatory compounds were applied to the somata of dorsal horn neurons by pressure ejection from micropipettes with tip diameters of 4 to $6 \mu \mathrm{m}$. Compounds to be applied by pressure ejection were added to standard recording medium without serum and with the $\mathrm{pH}$ adjusted with $\mathrm{HCl}$ or $\mathrm{NaOH}$. In rontrol experiments, pressure ejection of medium alone, with or without serum, produced negligible effects on membrane input resistance and resting potential. The ionic composition of the medium was changed and acidic amino acid antagonists were introduced by means of a continuous superfusion system. The standard recording medium contained, in millimolar concentration: $\mathrm{KCl}, 5.36 ; \mathrm{KH}_{2} \mathrm{PO}_{4}, 0.44$; $\mathrm{NaCl}, 136.9$; glucose, $50,1 \%$ heal-inactivated rat serum; $0.2 \%$ phenol red $\left(\mathrm{pH} \mathrm{7.2)} . \mathrm{CaCl}_{2}\right.$ and $\mathrm{MgCl}_{2}$ were included at various concentrations. Cultures were superfused with standard recording medium containing either $3 \mathrm{~mm}$
$\mathrm{Ca}^{2+}$ and $0.9 \mathrm{mM} \mathrm{Mg}^{2+}$ or $5 \mathrm{mM} \mathrm{Ca}^{2+}$ and $3 \mathrm{mM} \mathrm{Mg}^{2+}$ to inhibit polysynaptic potentials (see "Results")

The use of extracellular electrodes to stimulate the presynaptic neurons did not allow us to monitor the presynaptic action potential of individual DRG neurons. To provide evidence that successive stimuli resulted in the activation of the identical set of presynaptic DRG neurons, the stimulus strength was varied until a range was found in which large changes in the stimulus strength resulted in no change in the amplitude of the EPSP. The stimulus strength was then confined to the middle of the range for the duration of the dorsal horn neuron impalement. The EPSP was closely moritored througtioul the experiment. If sudden changes in EPSP amplitude were detected, the stimulus strength was adjusted and the experiment was restarted. If there was any ambiguity in the constancy of stimulation, the recording was ended. Most EPSPS studied were composed of one to four components, each evoked at a different but constant stimulus strength (see Fig. 4). Changes in the stimulus strength at which individual components were elicited were tolerated only when alterations in the total divalent cation concentration were made. After such a change, prolonged control records were obtained in order to ensure stability before proceeding with the experiment.

Chemicals were obtained from the following sources: L-glutamate and Laspartate (Sigma Chemical Co. and Grand Island Biological Co.); quisqualate, kainate, and 2-amino-4-phosphonobutyrate (Cambridge Research Biochemicals); 2-amino-5-phosphonovalerate (Sigma and Cambridge Research Biochemicals); and kynurenate (Sigma and Aldrich Chemical Corp.).

\section{Results}

Intracellular recordings were obtained from 64 dorsal horn neurons that received monosynaptic DRG input. No significant variation was found in the properties of neurons obtained from different platings and from cultures maintained 4 to 12 weeks in vitro. In low concentrations of divalent cations ( $3 \mathrm{~mm} \mathrm{Ca}^{2+}, 0.9 \mathrm{~mm} \mathrm{Mg}^{2+}$ ), spontaneous postsynaptic potentials were detected in more than $95 \%$ of dorsal horn neurons. Since no spontaneous synaptic activity or action potentials were detected when intracellular recordings were obtained from DRG neurons in the same cultures, spontaneous EPSPs were probably due to input from other dorsal horn neurons. Spontaneous synaptic activity was blocked by the addition of $\mathrm{Cd}^{2+}$ (100 to 200 $\mu \mathrm{M})$ or $\mathrm{Co}^{2+}(2$ to $5 \mathrm{~mm}$ ), or by increasing the total divalent cation concentration ( $5 \mathrm{mM} \mathrm{Ca}^{2+}$ and $3 \mathrm{mM} \mathrm{Mg}^{2+}$ ).

Electrical stimulation of DRG explants evoked EPSPs in more than $80 \%$ of dorsal horn neurons located within $1 \mathrm{~mm}$ of the explant. In recording medium containing $3 \mathrm{mM} \mathrm{Ca}^{2+}$ and $0.9 \mathrm{mM} \mathrm{Mg}^{2+}$, the postsynaptic response was multiphasic and prolonged (Fig. 1A), suggesting that at least part of the response was mediated by polysynaptic circuits. Two properties of the evoked synaptic potentials were used to discriminate between monosynaptic and polysynaptic EPSPs. (1) Later components of the EPSP did not follow repetitive stimulation at frequencies that caused only moderate

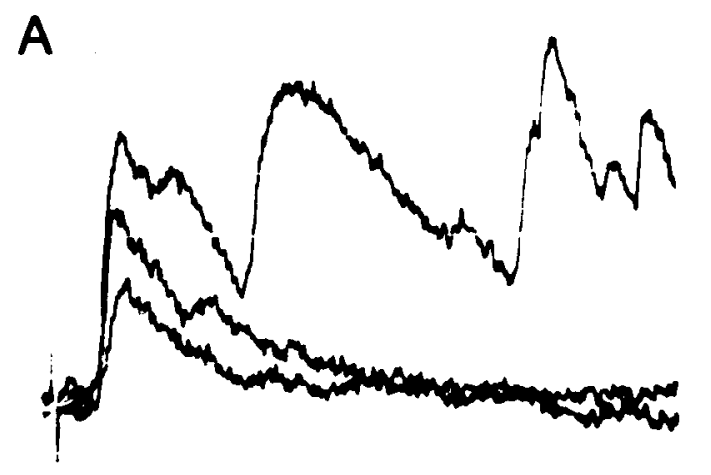

B

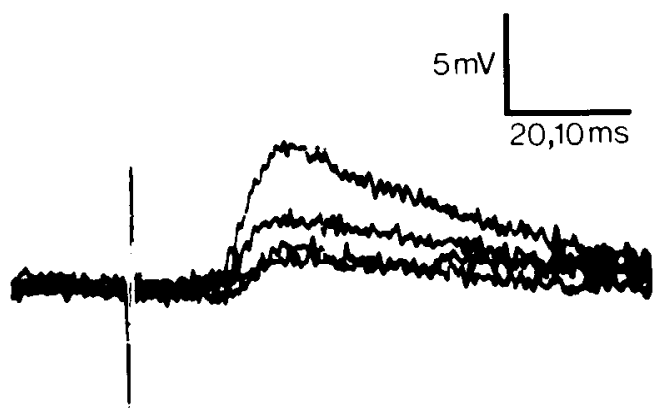

Figure 1. Effect of stimulation frequency and divalent cation concentration on mono- and polysynaptic EPSPs recorded in dorsal horn neurons after DRG explant stimulation. A, EPSPS evoked in a dorsal horn neuron by electrical stimulation of a DRG explant. Three stimuli were applied at $1 \mathrm{~Hz}$. Note that the later components disappeared with the second and third stimuli. The recording medium contained $3 \mathrm{mM} \mathrm{Ca}^{2+}$ and $0.9 \mathrm{mM} \mathrm{Mg}$. $B$, EPSPS evoked in the same cell by four stimuli $(1 \mathrm{~Hz})$ after switching to medium containing $5 \mathrm{mM} \mathrm{Ca}^{21}$ and $3 \mathrm{~mm} \mathrm{Mg}^{24}$. Resting potential $=-65 \mathrm{mV}$. Calibration is: $A, 5 \mathrm{mV} \times 20$ msec; $B, 5 \mathrm{mV} \times 10 \mathrm{msec}$ 

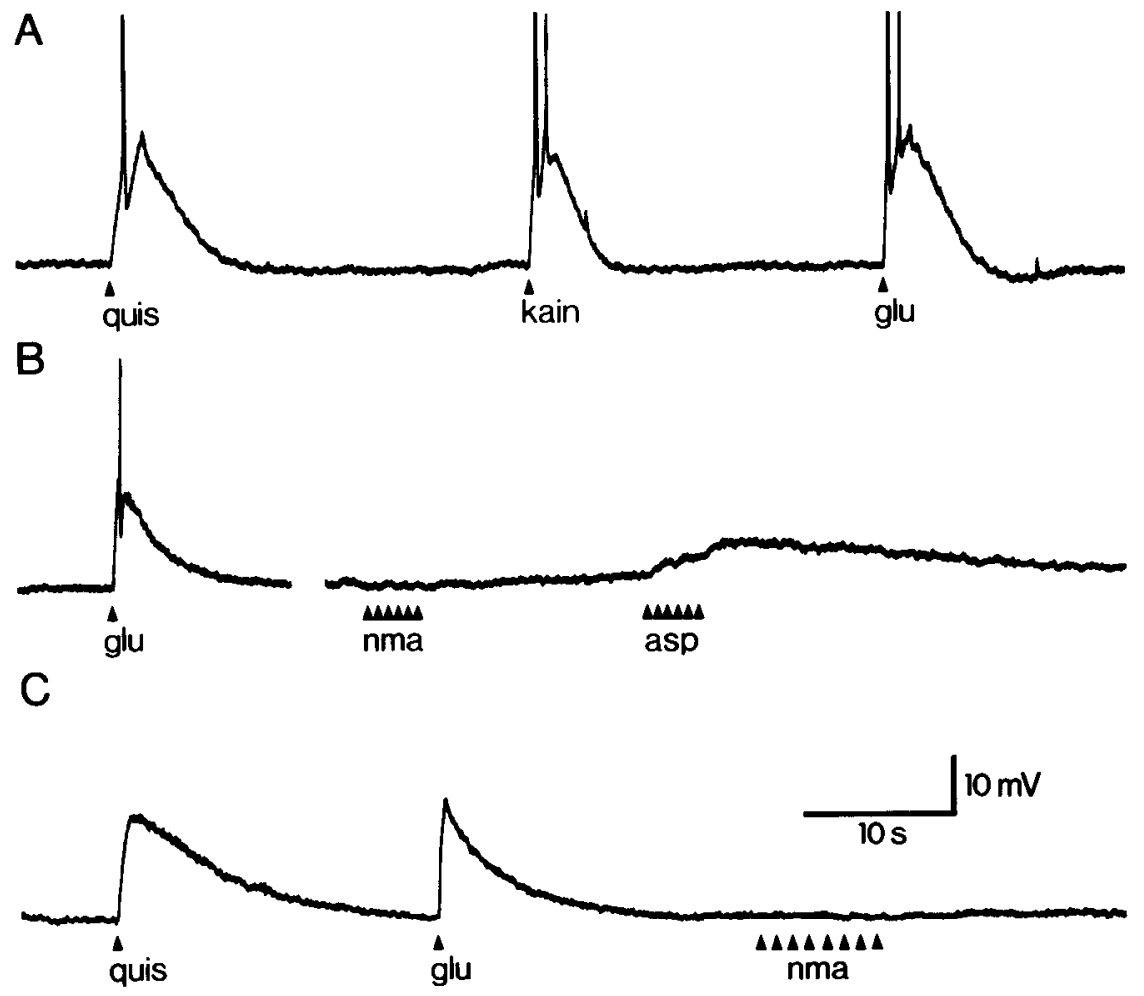

Figure 2. Effects of acidic amino acids and structural analogues on dorsal horn neurons. $A$, Quisqualate (quis: $0.1 \mathrm{sec} \times 5 \mu \mathrm{M})$, kainate (kain; $1 \mathrm{sec} \times 20 \mu \mathrm{M})$, and $\mathrm{L}$ glutamate ( $g / u ; 1 \mathrm{sec} \times 20 \mu \mathrm{M}$ ) were applied to the dorsal horn neuron (at the arrowheads) by pressure ejection from a pipette positioned within $30 \mu \mathrm{m}$ of the soma. Resting potential $=-60 \mathrm{mV}$. $B$, Responses of a different dorsal horn neuron to L-glutamate $(0.5 \mathrm{sec} X$ $20 \mu \mathrm{M}), N$-methyl-DL-aspartate ( $n \mathrm{ma} ; 1 \mathrm{sec} \times 200 \mu \mathrm{M})$, and L-aspartate (asp; $1 \sec \times 200 \mu \mathrm{M})$. Both L-aspartate and $\mathrm{N}$-methyl-DL-aspartate were applied six times as indicated by the arrowheads. Resting potential $=-65$ $\mathrm{mV}$. C, Responses of another dorsal horn neuron to pressure application of quisqualate $(1 \sec \times 2 \mu \mathrm{M})$, Lglutamate $(0.5 \mathrm{sec} \times 20 \mu \mathrm{M})$, and $N$-methyl-DL-aspartate (1 $\sec \times 200 \mu \mathrm{M} \times 8$ applications). Resting potential = $-63 \mathrm{mV}$. Drugs were ejected at 1 to $2.5 \mathrm{psi}$. Recording medium contained $5 \mathrm{mM} \mathrm{Ca}^{2+}$ and $3 \mathrm{mM} \mathrm{Mg}^{2+}$ in $A$ to $\mathrm{C}$ and in all subsequent figures.
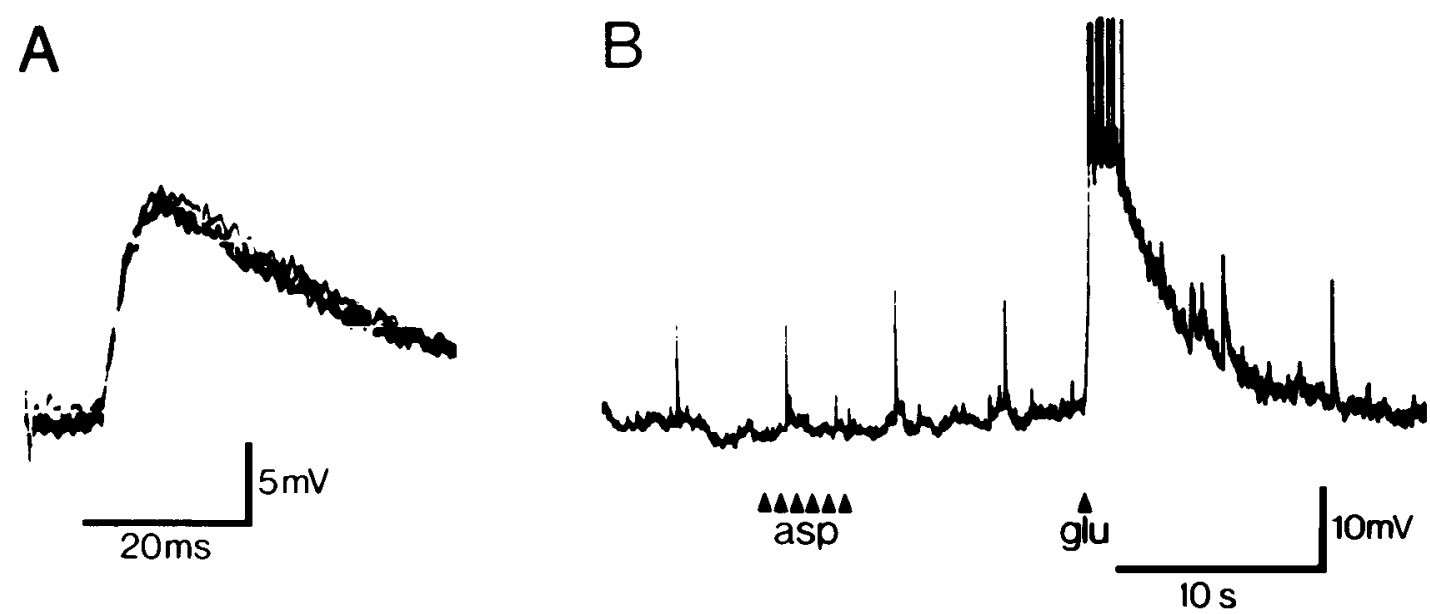

Figure 3. Monosynaptic sensory neuron input in the absence of L-aspartate sensitivity. The first record shows oscilloscope traces of four superimposed EPSPS. The second record is a chart record obtained from the same neuron, showing the responses to pressure application of L-aspartate (asp; 1 sec $X$ $200 \mu \mathrm{M} ; 6$ applications) and of L-glutamate (g/u; $1 \mathrm{sec} \times 20 \mu \mathrm{M})$. The fast upward deflections occurring at 5 -sec intervals are the evoked EPSPs shown at a faster time base in $A$. Resting potential $=-64 \mathrm{mV}$.

depression of the first component (Fig. 1A). (2) High concentrations of divalent cations ( $5 \mathrm{mM} \mathrm{Ca}^{2+}$ and $3 \mathrm{mM} \mathrm{Mg}^{2+}$ ) decreased or blocked all but the earliest, monophasic EPSP (Fig. 1B). The effect of divalent cations presumably reflects an increase in the spike threshold of all neurons in the culture (Frankenhaeuser and Hodgkin, 1957; Yellen, 1984) and a consequent blockade of polysynaptic pathways. DRG-evoked EPSPS were therefore classified as monosynaptic if their latencies were constant, if they followed $10 \mathrm{~Hz}$ stimulation, and if, in high levels of divalent cations, repetitive stimulation resulted in a monophasic EPSP. Depolarizations of slow onset or prolonged duration were not observed in dorsal horn neurons after stimulation of DRG explants, even at stimulus frequencies of up to $5 \mathrm{~Hz}$ for periods up to $8 \mathrm{~min}$.

The sensitivity to excitatory amino acids and related compounds was only tested in those dorsal horn neurons that received monosynaptic sensory input. The effects of excitatory amino acids and their analogues on dorsal horn neurons were consistent in all plat- ings. L-Glutamate (10 to $20 \mu \mathrm{M})$, quisqualate (1 to $10 \mu \mathrm{M})$, and kainate $(10$ to $20 \mu \mathrm{M})$ all were potent excitants. Kainate and Lglutamate were approximately equipotent, whereas quisqualate was 10 to 20 times more potent. These agonists caused rapid depolarizations of 10 to $40 \mathrm{mV}$ in 55 of 57 neurons. The depolarizations were often suprathreshold (Fig. 2). In contrast, NMDA, at concentrations up to $200 \mu \mathrm{M}$, had no effect (25 of 25 neurons). L-Aspartate (100 to $200 \mu \mathrm{M}$ ) either had no effect (22 of 25 neurons) or elicited small depolarizations ( 1 to $5 \mathrm{mV}$ ) after repeated application ( 3 of 25 neurons). However, all dorsal horn neurons that were insensitive to L-aspartate received monosynaptic input from DRG neurons (Fig. 3).

To determine whether amino acids, or compounds acting at amino acid receptors on dorsal horn neurons, might be transmitters at sensory synapses, we examined the effect of several antagonists of excitatory amino acid-evoked responses on the DRG-evoked monosynaptic EPSP recorded from dorsal horn neurons. Kynurenate 
A

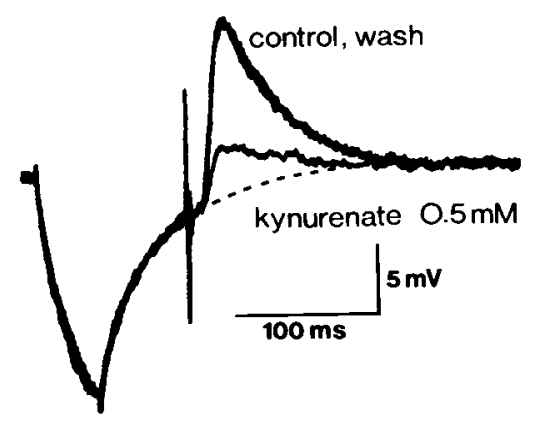

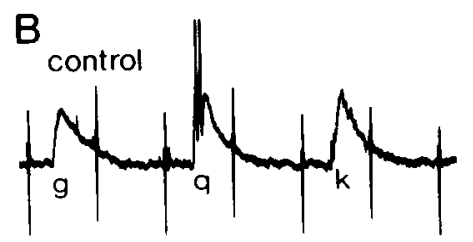

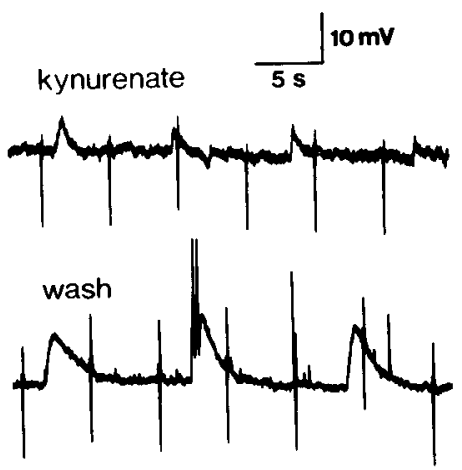

C

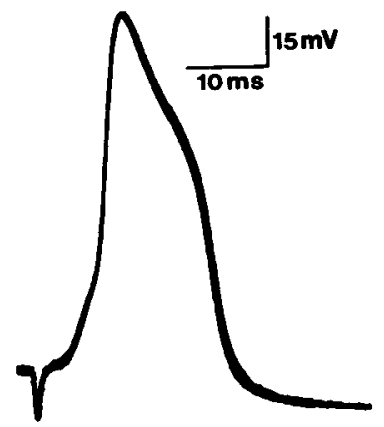

Figure 4. Effects of kynurenate on dorsal horn and DRG neurons. A, Responses of a dorsal horn neuron to a hyperpolarizing current pulse injected through the recording electrode and stimulation of a nearby DRG explant before, during, and after superfusion of $0.5 \mathrm{~mm}$ kynurenate. Sixteen responses were averaged in each condition and then superimposed. $B$. Chart records obtained from the same neuron shown in $A$, during which excitatory amino acids were applied by pressure ejection. The responses to L-glutamate $(g ; 1 \mathrm{sec} \times 20 \mu \mathrm{m} 0$, quisqualate $(q ; 0.2 \mathrm{sec} \times 2 \mu \mathrm{M})$, and $\mathrm{kainate}(\mathrm{k}$; $1 \mathrm{sec} \times 20 \mu \mathrm{M})$ were greatly attenuated by kynurenate $(0.5 \mathrm{~mm})$. Drugs were ejected at 1 psi. The fast deflections occurring at 5 -sec intervals are the evoked EPSPs and hyperpolarizing conductance pulses shown at a faster time base in $A$. Resting potential $=-65 \mathrm{mV}$. C, Action potentials recorded from a DRG neuron before, during, and after superfusion of $0.5 \mathrm{~mm}$ kynurenate. Sixteen action potentials were averaged in each condition and then superimposed. Action potentials were evoked by stimulation of the explant of which this neuron was a peripheral member. In this neuron potentials appeared to be generated by antidromic invasion, since there was a distinct delay from the stimulus artifact (stimulus duration was $200 \mu \mathrm{sec}$ ) and since the action potential could be "fractionated" into an initial segment spike by hyperpolarizing the neuron (not shown). Resting potential $=-75 \mathrm{mV}$.

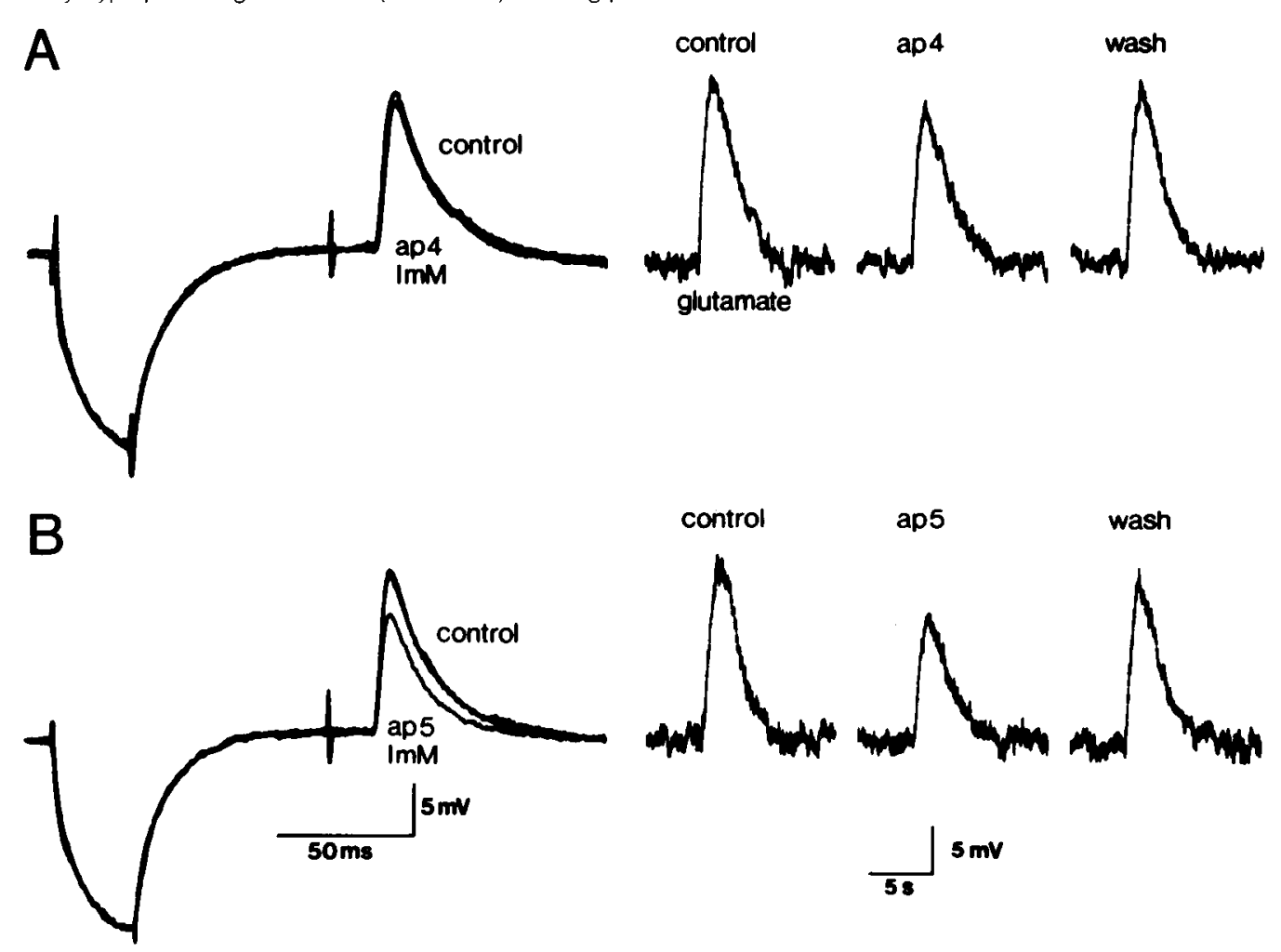

Figure 5. Effects of 2-amino-4 phosphonobutyrate and APV on synaptic and L-glutamate potentials of a dorsal horn neuron. $A$, The first trace shows averaged and superimposed responses to an intracellular current pulse and to stimulation of an adjacent DRG explant, before and during superfusion of 2-amino-4-phosphonobutyrate $(a p 4 ; 1 \mathrm{~mm})$. The next three traces are chart records of the responses to identical pressure ejections of L-glutamate $(1 \mathrm{sec} \times 20 \mu \mathrm{m})$ before, during, and after superfusion with 2-amino-4-phosphonobutyrate. $B$, The same neuron and experimental paradigm as shown in $A$ except that APV $(a p 5 ; 1 \mathrm{~mm})$ was superfused. The traces of EPSPS are averages of 16 responses in each condition. Resting potential $=-60 \mathrm{mV}$.

was the most potent antagonist of both the EPSP and the depolarization evoked by pressure ejection of L-glutamate, quisqualate, and kainate (Fig. 4, $A$ and $B$ ). Kynurenate at a concentration of $1 \mathrm{~mm}$ completely blocked the EPSP and the amino acid-evoked responses (data not shown). In contrast, neither 2-amino-4-phosphonobutyrate (Fig. 5A) nor L-glutamate diethylester at concentrations up to $1 \mathrm{~mm}$ significantly affected the DRG-evoked EPSP or the responses of dorsal horn neurons to L-glutamate (Fig. 5A), kainate, or quisqualate. At the same concentration, the NMDA receptor antagonist, APV, slightly reduced the amplitude of the EPSP and of the L-glutamateevoked depolarization (Fig. 5B). Piperidine dicarboxylate and $\gamma$-Dglutamylglycine reduced the amplitude of the EPSP and the Lglutamatc cvoked depolarizations, but with a potency less than half that of kynurenate (not shown). D-Tubocurarine, hexamethonium, and dihydro- $\beta$-erythroidine (all $10 \mu \mathrm{M}$ ), which have no reported ability to antagonize amino acid-evoked responses, had no effect on the amplitude of the DRG-evoked EPSP. In addition, a series of compounds related to kynurenate was tested for both agonist and 
A

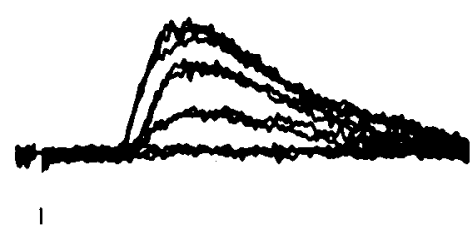

B

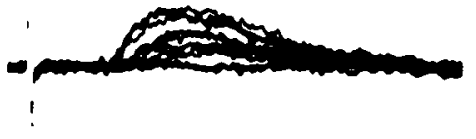

C

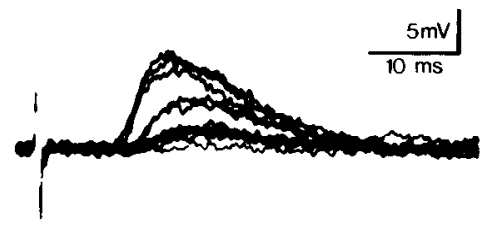

Figure 6 . The effect of kynurenate on EPSPs evoked by multiple DRG inputs. $A, B$, and $C$ are EPSPs recorded from a dorsal horn neuron before, during, and after washout of $0.5 \mathrm{~mm}$ kynurenate, respectively. EPSPs were evoked by electrical stimulation of a nearby DRG explant at three distinct stimulus strengths. A complete recovery from kynurenate was not achieved. All records are unaveraged responses photographed directly from an oscilloscope. Resting potential $=-66 \mathrm{mV}$.

A

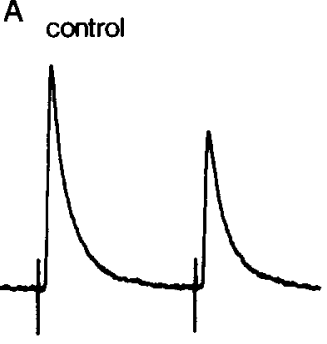

B $\mathrm{Co}^{2+}$

D control

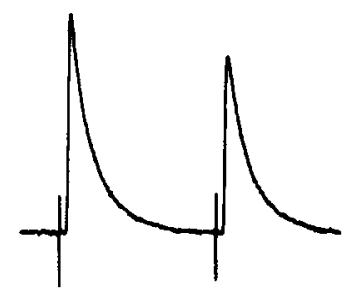

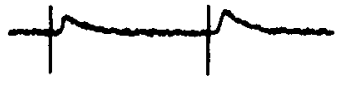

E

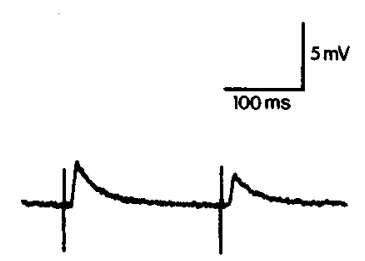

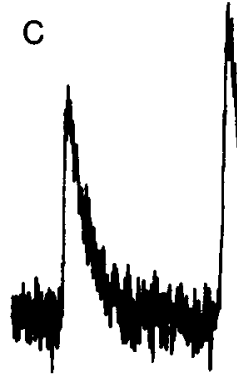

$\mathbf{F}$

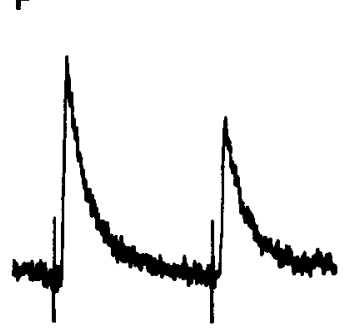

Figure 7. The effects of kynurenate and $\mathrm{Co}^{2+}$ on synaptic depression at DRG-dorsal horn synapses. $A$, Control records of EPSPs evoked in dorsal horn neurons by two stimuli applied to the explant at 200msec intervals. $B, E P S P$ s resulting from stimulation parameters identical to those shown in $A$ after the introduction of $2 \mathrm{mM} \mathrm{Co}^{2+}$ to the superfusate. $C$, Same record as in $B$ at $\times 12$ gain to show that, when the first EPSP in the pair is scaled to match that of control (in A), the amplitude of the second EPSP is greatly potentiated. $D$, Control record after washout of $\mathrm{Co}^{2+}$. E, EPSPs resulting from stimulation identical to that in $A$ in the presence of $0.5 \mathrm{~mm}$ kynurenate. $F$, Same record as in $E$ at $\times 5$ gain. All traces are averages of 16 records. Resting potential $=-65 \mathrm{mV}$. The calibration in $E$ applies to $A, B, D$, and $E$.

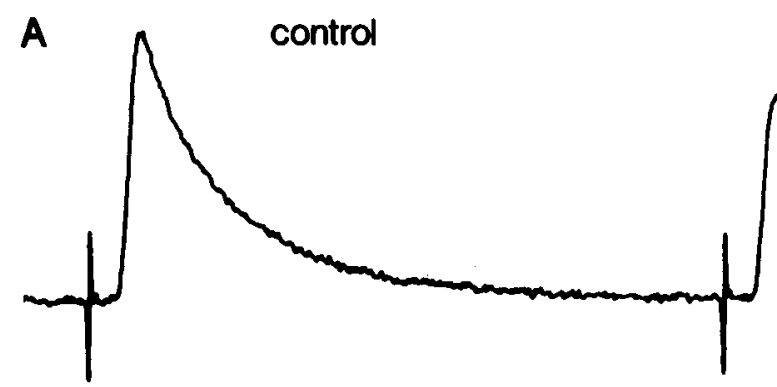

B kymurenate
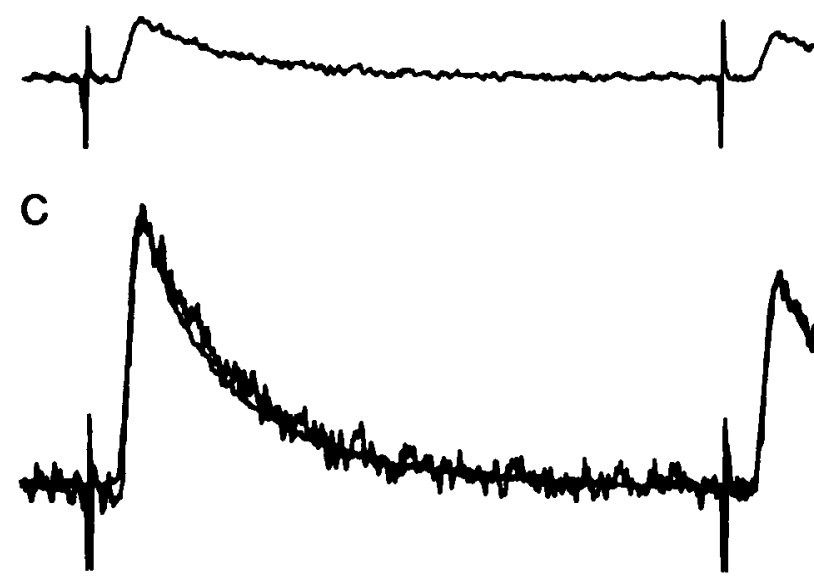

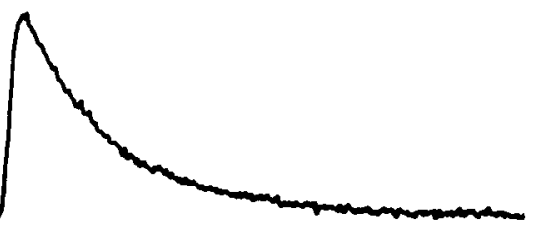

$4 \mathrm{mV}$

$50 \mathrm{~ms}$

Figure 8. The effect of kynurenate on synaptic depression and EPSP time course. $A$, EPSPs evoked in a dorsal horn neuron after two DRG explant stimuli applied at 250 msec intervals. $B$, EPSPs evoked by stimulation parameters identical to those shown in $A$ after the introduction of $0.5 \mathrm{~mm}$ kynurenate to the superfusate. $C$, Superimposition of traces after increasing the gain such that the amplitude of the first EPSP in $B$ is equal to that in record $A$. Each trace is the average of 16 records. Resting potential $=$ $-65 \mathrm{mV}$. 


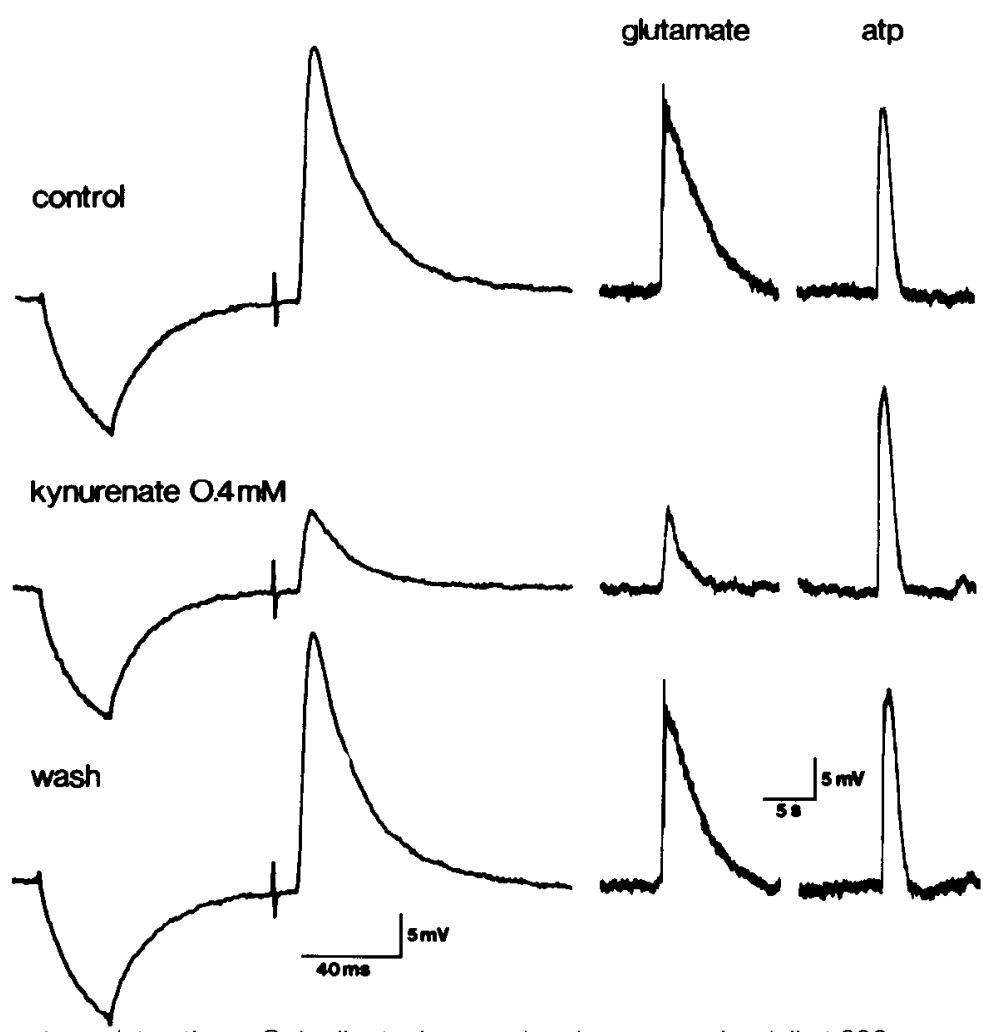

Figure 9. The effects of kynurenate on the responses of a dorsal horn neuron to DRG explant stimulation and pressure application of L-glutamate and ATP. The first traces in each row show the responses to an intracellular current pulse followed by stimulation of a nearby DRG explant. The next two traces are chart records of the responses to pressure ejection of L-glutamate $(1 \mathrm{sec} \times 20 \mu \mathrm{M})$ and ATP ( $1 \mathrm{sec}$ $\times 20 \mu \mathrm{M})$. The responses in the second row were recorded in the presence of $0.4 \mathrm{~mm}$ kynurenate. The responses in the third row were recorded after washing out kynurenate. Each EPSP trace is the average of 16 records. Resting potential $=-68 \mathrm{mV}$.

antagonist actions. Quinolinate, kynurenine, kynurenamine (all at 200 $\mu \mathrm{M})$, xanthurenate ( $1 \mathrm{~mm})$, and picolinate $(1 \mathrm{~mm}$ ) were all inactive.

Since kynurenate was the most potent antagonist at DRG-dorsal horn synapses identified in these studies, we performed additional experiments to determine its site and mechanism of action. The reduction by kynurenate of EPSP amplitude and of amino acidevoked depolarizations was not associated with any change in the input resistance of dorsal horn neurons (Fig. 4A; 10 neurons) or with changes in the threshold, amplitude, or duration of the action potential recorded intracellularly from the cell bodies of DRG neurons (Fig. 4C; 4 neurons). All EPSPS, whether spontaneous or recorded in response to DRG stimulation, were antagonized by kynurenate (22 neurons).

The blockade of amino acid-evoked depolarizations of dorsal horn neurons clearly demonstrates a postsynaptic site of action of kynurenate. However, it is possible that kynurenate antagonized the EPSP by blocking action potential propagation in the axons of DRG neurons presynaptic to dorsal horn neurons. Most of the EPSPS studied were composed of smaller units which could be separated by a continuous gradation in the stimulus strength. Graded stimulation revealed discrete steps in the amplitude of the EPSP (Fig. 6) and suggested that several DRG neurons can form functional synapses on the same dorsal horn neuron. In the presence of kynurenate the same number of discrete steps could be evoked, although the amplitude of each was reduced to a similar extent. Moreover, recruitment of each step in the EPSP occurred at a stimulus strength identical to that required before addition of kynurenate. These findings indicate that kynurenate did not block spike propagation in DRG axons and are consistent with a postsynaptic site of inhibition of the DRG-evoked EPSP.

The site and mechanisms of action of kynurenate were further investigated by examining their effect on synaptic depression evoked by paired presynaptic stimuli. At stimulus intervals of 50 to $400 \mathrm{msec}$ the second EPSP was depressed to 50 to $70 \%$ of the first EPSP in medium containing $5 \mathrm{mM} \mathrm{Ca}^{2+}$ and $3 \mathrm{mM} \mathrm{Mg}^{2+}$ (Fig. $7 A$ ). When the $\mathrm{Mg}^{2+} / \mathrm{Ca}^{2+}$ ratio was increased or $\mathrm{Ca}^{2+}$ channel blockers such as $\mathrm{Co}^{2+}$ or $\mathrm{Cd}^{2+}$ were added to the superfusion medium, the depression was either partly or entirely abolished $(n=$ 6). In experiments in which the first EPSP was substantially de- creased, a potentiation of the second EPSP resulted (Fig. 7, $B$ and C). In contrast, when the first EPSP was decreased to about $20 \%$ of its control amplitude by addition of $0.5 \mathrm{~mm}$ kynurenate, the second response was decreased to a similar extent (Fig. 7, $E$ and $F$ ). Moreover, the addition of kynurenate sometimes resulted in an enhancement of the depression. In control conditions in the experiment illustrated in Figure $7 D$, the ratio of the amplitudes of the second to the first EPSP was 0.8, whereas in the presence of kynurenate (Fig. $7, E$ and $F$ ) the ratio was decreased to 0.74 , indicating that the depression of the second EPSP was increased. The mean increase in depression observed in the presence of kynurenate was $13 \%$ (range $-2 \%$ to $44 \% ; n=7$; in Fig. 7 , depression increased $8 \%$ ). The enhancement of depression could be due to a slight use dependency of the kynurenate block. During trains of single stimuli the amplitude of the EPSP reached steady state after one to five repetitions. Comparison of the steady-state amplitudes evoked at $0.2 \mathrm{~Hz}$ and $10 \mathrm{~Hz}$ in the presence and absence of kynurenate revealed that the antagonism was enhanced approximately 1.2-fold at the higher frequency. Other possible mechanisms underlying the enhanced depression are discussed beiow. It is important to note that the reduction in EPSP amplitude produced by kynurenate was not invariably associated with an enhancement of synaptic depression in the paired stimulus experiments. Figure 8 illustrates the result of an experiment in which the degree of depression was unaltered by kynurenate. This indicated that the ability of kynurenate to block sensory neuron synapses was independent of its action to enhance synaptic depression.

Although kynurenate antagonized the response of dorsal horn neurons to L-glutamate, quisqualate, and kainate, it did not inhibit the response of dorsal horn neurons to all excitatory transmitter candidates. We have shown previously that ATP excites a subpopulation of dorsal horn neurons by activating a membrane conductance similar to that evoked by L-glutamate (Jahr and Jessell, 1983). The reversal potential of both L-glutamate (Mayer and Westbrook, 1984)- and ATP (C. E. Jahr, unpublished observations)-evoked responses on spinal neurons is near $0 \mathrm{mV}$. Superfusion of dorsal horn neurons with a concentration of kynurenate sufficient to antagonize both the DRG-evoked EPSP and the response to L-glutamate had no effect on the response of the same neuron to ATP (Fig. 9). 


\section{Discussion}

Several groups have used dissociated cultures of DRG and spinal cord neurons to examine synaptic transmission at DRG-spinal cord, spinal cord-spinal cord, and spinal cord-DRG synapses (Ransom et al., 1977a; Choi and Fischbach, 1981, MacDonald et al., 1983). We have found that one major difficulty in studying primary afferent transmission between DRG and dorsal horn neurons in dissociated cell co-cultures is the low probability of detecting synaptically coupled pairs of neurons. In the present study we overcame this problem by growing explants of DRG neurons in co-culture with dissociated dorsal horn neurons. By stimulating many DRG neurons simultaneously, we were able to increase, dramatically, the probability of recording from dorsal horn neurons that could be excited monosynaptically. The high density of DRG processes near the explant may also have increased the frequency of synaptic contacts between DRG and dorsal horn neurons.

Several criteria were used to provide evidence that synaptic potentials recorded from dorsal horn neurons after extracellular stimulation of DRG were monosynaptic. As previously described (MacDonald et al., 1983) the divalent cation concentration in the recording medium was increased to a level sufficient to block spontaneous synaptic input to dorsal horn neurons. In this condition, monosynaptic EPSPs generated in dorsal horn neurons by DRG stimulation could be studied without contamination from dorsal hornspinal neuron interactions. If the evoked EPSPs followed repetitive stimulation of $10 \mathrm{~Hz}$, appeared with a constant latency, and were monophasic, they were classified as monosynaptic. The high proportion of dorsal horn neurons that received DRG input under these recording conditions enabled us to compare the pharmacology of monosynaptic FPSPs with that of the potentials evoked by various excitatory amino acid transmitter candidates on the same dorsal horn neurons.

The elevation of the $\mathrm{Mg}^{21}$ concentration in the recording medium was designed to discriminate between L-aspartate and L-glutamate as potential sensory transmitter candidates. The ion channel of mammalian central neurons that is sensitive to both $L$-aspartate and L-glutamate and is activated selectively by the L-aspartate analogue, NMDA, is subject to a voltage-dependent blockade by micromolar concentrations of $\mathrm{Mg}^{2+}$ (Mayer et al., 1984; Nowak et al., 1984). Under our recording conditions, in the presence of $3 \mathrm{mM} \mathrm{Mg}^{2+}$, this receptor/channel complex is likely to be inactive. The failure of NMDA and L-aspartate to excite dorsal horn neurons appears to confirm this possibility. The selective depolarization observed by Lgluatamate under these ionic conditions therefore suggests that $L$ glutamate but not L-aspartate may mediate the fast afferent EPSP recorded from dorsal horn neurons. The weak antagonist action of APV on DRG-evoked EPSPs further suggests that if L-glutamate is the transmitter at these symapses, it is urlikely to mlediale its effects via the NMDA receptor subtype, at least at a potential range near rest. From these studies, however, we cannot exclude the possibility that L-aspartate is a sensory transmitter at synapses that cannot be detected under these recording conditions.

The use of kynurenate has provided further evidence that $L$ glutamate or a closely related compound is the transmitter at the sensory neuron synapses examined in these experiments. The blockade by kynurenate of L-glutamate responses of dorsal horn neurons at concentrations that also reduced or abolished the DRGevoked monosynaptic EPSP is consistent with the idea that Lglutamate is a primary sensory transmitter. However, with extracellular stimulation of DRG neurons, the presynaptic spike could not be monitored to ensure that kynurenate was not altering spike threshold or propagation in those DRG neurons that synapsed onto the dorsal hom neuron under study. This possituility seems urilikely for several reasons. The amplitude of the EPSP decreased gradually as kynurenate was introduced into the bath. Blockade of the presynaptic spike would be expected to cause an abrupt decrease or blockade of the EPSP. In dorsal horn neurons where the EPSP was evoked by multiple DRG inputs (distinguished by gradations in stimulus strength), each EPSP component was decreased by kynurenate without a reduction in the number of components recorded. The stimulus strengths at which each successive increment occurred under control conditions were not altered by addition of kynurenate. EPSPs which were all or none in their activalion (suggesling a single input) were also decreased in a continuous manner as kynurenate was introduced into the recording chamber. Furthermore, kynurenate did not alter the threshold or duration of the action potential, assessed by intracellular recordings from randomly selected DRG neurons within the explant.

To distinguish between a presynaptic and postsynaptic site of action of kynurenate, we also used a method that should be sensitive to changes in the amplitude and duration of the spike at DRG axon terminals. This method is based on studies of the phenomena of synaptic depression and potentiation at the neuromuscular junction. Thies (1965) has reported that in normal or elevated levels of $\mathrm{Ca}^{2+}$, synaptic depression results from a decrease in the amount of transmitter released. Thus, the quantal content of the second endplate potential (EPP) evoked by a pair of closely spaced stimuli is lower than that of the first EPP. The magnitude of the depression is a function of the number of quanta released by the first stimulus in that, when the quantal content of the first EPP is reduced by decreasing the $\mathrm{Ca}^{2+} / \mathrm{Mg}^{2+}$ ratio, a potentiation rather than a depression of the second EPP occurs. It has been suggested that depression results when the amount of transmitter released by the first stimulus is sufficient to deplete the store of transmitter available for release by the second stimulus. $d$-Tubocurarine, a postsynaptic receptor antagonist, decreases the amplitude of all EPPs to the same extent (Otsuka et al., 1962), indicating that antagonists acting at postsynaptic receptors do not alter synaptic depression.

We observed similar phenomena at sensory neuron synapses. In recording medium containing $5 \mathrm{mM} \mathrm{Ca}^{2+}$ and $3 \mathrm{mM} \mathrm{Mg}^{2+}$, the EPSP recorded in dorsal horn neurons in response to the second of a pair of presynaptic DRG stimuli was depressed. When transmitter release was decreased by a reduction in the $\mathrm{Ca}^{2+} / \mathrm{Mg}^{2+}$ ratio, or by addition of $\mathrm{Ca}^{2+}$ channel blockers to the recording medium, the degree of synaptic depression was diminished and, at times, when transmitter release was severely decreased, the EPSP was potentiated. Thus, presynaptic blockers reduce the extent of synaptic depression at sensory synapses. In contrast, kynurenate did not reduce the extent of synaptic depression. In fact, at some but not all sensory neuron synapses, the degree of depression was actually increased. At least four mechanisms could account for the enhancement of synaptic depression.

1. Kynurenate may block ion channels opened by the sensory transmitter. In this case the time constant of decay of the EPSP would be decreased, the antagonism would be use dependent, and, depending on the site of channel block in the potential field of the membrane, the block might be voltage dependent (Adams, 1976; Beam, 1976). The time constant of the decay of the EPSP was unaltered by kynurenate, although small changes in the decay phase might not be resolved in the present current clamp experiments. The voltage dependency of the blockade could not be studied with enough precision to test its possible contribution. The antagonism of the EPSP by kynurenate was use dependent. Use dependence, however, could also be due to a higher affinity of kynurenate for the activated receptor/channel complex. These observations suggest that channel block is not the primary mechanism by which kyruurenate exerts its antagonism of DRG-evoked EPSPS in dorsal horn neurons. Voltage clamp analysis of the underlying conductance change should provide a more direct measurement of the time course of the synaptic event.

2. The increased depression after addilion of kynurenale could result from nonlinearities in the membrane properties of dorsal horn neurons. Large EPSPs will depolarize neurons to such an extent that membrane rectification becomes an important factor in determining the amplutide of the EPSP. An enhancement of depression would 
be observed in the presence of concentrations of kynurenate which decrease EPSP amplitudes to the degree that membrane rectification is not an important factor. However, this explanation is unlikely to account for our results, since even when the control EPSPs were quite small (2 to $5 \mathrm{mV}$ ), an enhancement of depression was often observed. Membrane rectification is therefore unlikely to play a major role in increasing the degree of depression.

3. Similarly, nonlinear temporal summation of the components that make up the EPSP could account for the increased depression when the control EPSP was large. However, since the reversal potential for these EPSPs is probably near $0 \mathrm{mV}$ (MacDonald et al., 1983), EPSPs on the order of 2 to $5 \mathrm{mV}$ would not be affected significantly by nonlinear summation.

4. If clearance of transmitter from the synaptic cleft occurs at a rate such that individual transmitter molecules can bind sequentially to many receptors, the introduction of a receptor antagonist could accelerate transmitter clearance by blocking transmitter/receptor binding and thereby facilitating transmitter diffusion. Such a mechanism has been proposed to account for the decreased EPP decay time constant and, in part, for the decreased EPP amplitude by curare and $\alpha$-bungarotoxin at neuromuscular junctions treated with anticholinesterases (Katz and Miledi, 1973).

Of these mechanisms, use-dependent block and accelerated transmitter clearance could account for the enhanced depression that was sometimes observed in the presence of kynurenate. The reason for the variability of the enhanced depression is not known. It is possible that distinct populations of dorsal horn neurons express different classes of amino acid receptors/channels and that these are antagonized by kynurenate by multiple mechanisms. Regardless of the mechanisms underlying the enhancement of synaptic depression, the reduction of the amplitude of the DRG-evoked EPSP in the absence of enhancement of synaptic depression or change in EPSP time course indicates that blockade of synaptic transmission by kynurenate can occur independently of this action.

Our studies with amino acid agonists and antagonists provide strong evidence that L-glutamate, or a compound with similar affinity for the synaptic receptor, is the transmitter mediating the fast EPSP at sensory neuron synapses examined in this study. Biochemical studies demonstrating release of L-glutamate from the terminal regions of primary afferent fibers have provided some evidence that L-glutamate itself may be the transmitter released from sensory terminals (Roberts, 1974; Takeuchi et al., 1983). The high density of $\mathrm{L}$-glutamate receptor-binding sites in the superficial dorsal horn of the spinal cord has provided some further support for a role of Lglutamate in sensory transmission (Gireenamyre et al., 1984).

From our studies in culture, the proportion of DRG neurons that release L-glutamate-like compounds as primary afferent transmitters is not clear. It is possible that culture conditions have in some way restricted our analysis to a subpopulation of primary afferent synapses. Studies on newborn rat spinal cord preparations pertormed in parallel with the experiments described here, however, have demonstrated the ability of kynurenate to antagonize both la EPSPS and cutaneous input to dorsal horn neurons ( $K$. Yoshioka and C. E. Jahr, manuscripts in preparation), suggesting that primary afferents conveying diverse sensory modalities release L-glutamate-like compounds as primary sensory transmitters in the mammalian spinal cord. From our studics on the chemosensitivity of cultured dorsal horn neurons to pulative sensory transmitters, the only compounds known to have postsynaptic actions consistent with a fast excitatory transmitter role are the acidic amino acids and the nucleotide ATP (Jahr and Jessell, 1983). Our present results do not rule out the possibility that ATP may be a sensory transmitter released from a small proportion of primary afferent fibers. Studies on the chemosensitivity of dorsal horn neurons in vivo have established that neurons which are excited by low threshold $C$ fiber activation are preferentially activated by ATP (Fyffe and Perl, 1984). Future studies to correlate the sensory fiber types that use L-glutamate-like compounds as sensory transmitters should be possible with the recent identification of monoclonal antibodies that label surface antigens on functional subpopulations of DRG neurons in situ and in cell culture (Dodd et al., 1984; Jessell and Dodd, 1985).

\section{References}

Adams, P. R. (1976) Drug blockade of open end-plate channels. J. Physiol. (Lond.) 260: 531-552.

Ault, B., R. H. Evans, A. A. Francis, D. J. Oakes, and J. C. Watkins (1980) Selective depression of excitatory amino acid induced depolarizations by magnesium ions in isolated spinal cord preparations. J. Physiol. (Lond.) 307: $413-428$

Beam, K. G. (1976) A voltage clamp study of the effect of two lidocaine derivatives on the time course of end-plate currents. J. Physiol. (Lond.) 258: $279-300$

Choi, D. W., and G. D. Fischbach (1981) GABA-mediated synaptic potentials in chick spinal cord and sensory neurons. J. Neurophysiol. 45: 632-643.

Davies, J., and J. C. Watkins (1981) Differentiation of kainate and quisqualate receptors in the cat spinal cord by selective antagonism with gamma-D (and L)-glutamylglycine. Brain Res. 206: 172-177.

Davies, J., A. A. Francis, A. W. Joncs, and J. C. Watkins (1981a) 2.Aminophosphonovalerate (2APV), a potent and selective antagonist of amino acid-induced and synaptic excitation. Neurosci. Lett. 21: 77-81.

Davies, J., R. H. Evans, A. A. Francis, A. W. Jones, and J. C. Watkins (1981b) Antagonism of excitatory amino acid-induced and synaptic excitation of spinal neurons by cis-2,3-piperidine dicarboxylate. J. Neurochem. 36: 1305-1307.

Dodd, J., D. Solter, and T. M. Jessell (1984) Monoclonal antibodies against carbohydrate differentiation antigens identify subsets of primary sensory neurons. Nature 311: 469-472.

Eccles, J. C. (1984) The Physiology of Synapses, Springer-Verlag, New York.

Elmslie, K. S., and D. Yoshikami (1983) Effects of kynurenate on root potentials evoked by synaptic activity and amino acids in the frog spinal cord. Soc. Neurosci. Abstr. 9: 263.

Engberg, I., and K. C. Marshall (1979) Reversal potential for la excitatory post synaptic potentials in spinal motoneurons of cats. Neuroscience 4: 1583-1591.

Finkel, A. S., and S. J. Redman (1983) The synaptic current evoked in cat spinal motoneurons by impulses in single group 1a axons. J. Physiol. (Lond.) 342: 615-632.

Foster, A. C., and G. E. Fagg (1984) Acidic amino acid binding sites in mammalian neuronal membranes: The characteristics and relationship to synaptic receptors. Brain Res. Rev. 7: 103-164.

Francis, A. A., A. W. Jones, and J. C. Watkins (1980) Dipeptide antagonists of amino acid-induced and synaptic excitation in the frog spinal cord. J. Neurochem. 35: 1458-1460.

Frankenhaeuser, B., and A. L. Hodgkin (1957) The action of calcium on the electrical properties of squid dxons. J. Physiol. (Lond.) 137. 218-244.

Fyffe, R., and E. R. Perl (1984) Selective excitation of a subpopulation of cat dorsal horn neurons by ATP: Possible role as an excitatory sensory transmitter. Proc. Natl. Acad. Sci. U. S. A. 81: 6890-6893.

Ganong, A. H., T. H. Lanthorn, and C. W. Cotman (1983) Kynurenic acid inhibits synaptic and acidic amino acid-induced responses in the rat hippocampus and spinal cord. Brain Res. 273: 170-174.

Greenamyre, J. T., A. B. Young, and J. B. Penny (1984) Quantitative autoradiographic distribution of $\mathrm{L}\left[{ }^{3} \mathrm{H}\right] \mathrm{glutamate}$-hinding sites in rat central nervous system. J. Neurosci. 4: 2133-2144.

Jahr, C. E., and T. M. Jessell (1983) ATP excites a subpopulation of rat dorsal horn neurons. Nature 304: 730-733.

Jessell, T. M., and J. Dodd (1985) Structure and expression of differentiation antigens on functional subsets of primary sensory neurons. Philos. Trans. R. Soc. Lond. (Biol.) 308: 271-281.

Kalz, B., and R. Miledi (1973) The binding of acetylcholine to receptors and its removal from the synaptic cleft. J. Physiol. (Lond.) 231: 549-574

MacDonald, R. L., R. Y. K. Pun, E. A. Neale, and P. G. Nelson (1983) Synaptic interactions between mammalian central neurons in cell culture. I. Reversal potential for excitatory postsynaptic potentials. J. Neurophysiol. 49: $1428-1441$.

Mayer, M. L., and G. L. Westbrook (1984) Mixed agonist action of excitatory amino acids on mouse spinal cord neurons under voltage clamp. J. Physiol. (Lond.) 354: 29-53.

Mayer, M. L., G. L. Westbrook, and P. B. Guthrie (1984) Voltage-dependent block by $\mathrm{Mg}^{2+}$ of NMDA responses in spinal cord neurons. Nature 309 : 261-263. 
Nowak, L., P. Bregestovski, P. Ascher, A. Herbet, and A. Prochiantz (1984) Magnesium gates glutamate-activated channels in mouse central neurons. Nature 307: 462-465.

Otsuka, M., M. Endo, and Y. Nonomura (1962) Presynaptic nature of neuromuscular depression. Jpn. J. Physiol. 12: 573-583.

Perkins, M. N., and T. W. Stone (1982) An iontophoretic investigation of the actions of convulsant kynurenines and their interaction with the endogeneous excitant quinolinic acid. Brain Res. 247: 184-187.

Ransom, B. R., C. N. Christian, P. N. Bullock, and P. G. Nelson (1977a) Mouse spinal cord in cell culture. II. Synaptic activity and circuit behavior. J. Neurophysiol. 40: 1151-1162.

Ransom, B. R., P. N. Bullock, and P. G. Nelson (1977b) Mouse spinal cord in cell culture. III. Neuronal chemosensitivity and its relationship to synaptic activity. J. Neurophysiol. 40: 1163-1177.

Roberts, P. J. (1974) The release of amino acids with proposed transmitter function from the cuneate and gracile nuclei of the rat, in vivo. Brain Res. 67: $419-428$

Roberts, P. J., P. Keen, and J. F. Mitchell (1973) The distribution and axonal transport of free amino acids and related compounds in the dorsal sensory neuron of the rat, as detcrmincd by the dansyl reaction. J. Neurochem. 21: 199-209.

Salt, T. E., and R. G. Hill (1983) Neurotransmitter candidates of somatosensory primary alferent libers. Neuroscience 10: 1083-1103.

Takeuchi, A., K. Onodera, and R. Kawagoe (1983) The effects of dorsal root stimulation on the release of endogenous glutamate from the frog spinal cord. Proc. Jpn. Acad. 59: 88-92

Thies, R. E. (1965) Neuromuscular depression and the apparent depletion of transmitter in mammalian muscle. J. Neurophysiol. 28: 427-442.

Urban, L., and M. Randic (1984) Slow excitatory transmission in rat dorsal horn: Possible mediation by peptides. Brain Res 290: 336-341.

Watkins, J. C., and R. H. Evans (1981) Excitatory amino acid transmitters. Annu. Rev. Pharmacol. Toxicol. 21: 165-204.

Yamamoto, M., H. M. W. Steinbusch, and T. M. Jessell (1981) Differentiated properties of identificd scrotonin ncurons in dissociated cultures of embryonic rat brain stem. J. Cell Biol. 91: 142-152.

Yellen, G. (1984) lon effects on ion channels. In 1984 Short Course Syllabus: Molecular Mechanisms of Signaling Across Neuronal Membranes, pp. 11-20, Society for Neuroscience, Washington, D.C. 